\title{
Effects of a Thermal Agent and Physical Activity on Muscle Tendon Stiffness, as Well as the Effects Combined With Static Stretching
}

\section{AUTHOR(S):}

Fujita, Kosuke; Nakamura, Masatoshi; Umegaki, Hiroki; Kobayashi, Takuya; Nishishita, Satoru; Tanaka, Hiroki; Ibuki, Satoko; Ichihashi, Noriaki

\section{CITATION:}

Fujita, Kosuke ... [et al]. Effects of a Thermal Agent and Physical Activity on Muscle Tendon Stiffness, as Well as the Effects Combined With Static Stretching. Journal of sport rehabilitation 2018, 27(1): 66-72

\section{ISSUE DATE:}

2018-01

\section{URL:}

http://hdl.handle.net/2433/243864

\section{RIGHT:}

Accepted author manuscript version reprinted, by permission, from 'Journal of sport rehabilitation' 2018, 27(1): 66-72, https://doi.org/10.1123/jsr.2015-0165. (c) Human Kinetics, Inc.; This is not the published version. Please cite only the published version.; こ の論文は出版社版でありません。引用の際には出版社版をご確認ご利用ください。 
1 Effects of a Thermal Agent and Physical Activity on Muscle Tendon Stiffness, as Well

2 as the Effects Combined With Static Stretching.

4 Kosuke Fujita ${ }^{1,2}$, Masatoshi Nakamura, PhD $^{1,3}$, Hiroki Umegaki ${ }^{1}$, Takuya Kobayashi ${ }^{1}$,

5 Satoru Nishishita ${ }^{1,4,5}$, Hiroki Tanaka ${ }^{1,6}$, Satoko Ibuki $^{1}$, Noriaki Ichihashi, PhD $^{1}$

6 1Human Health Sciences, Graduate School of Medicine, Kyoto University, Kyoto, Japan

$7 \quad$ 2Department of Rehabilitation, Nagoya University Hospital, Aichi, Japan

$8 \quad$ IInstitute for Human Movement and Medical Sciences, Niigata University of Health and

9 Welfare, Niigata, Japan

104 Institute of Rehabilitation Science, Tokuyukai medical corporation, Osaka, Japan

$11{ }^{5}$ Kansai Rehabilitation Hospital, Tokuyukai medical corporation, Osaka, Japan

$12 \quad{ }^{6}$ Rehabilitation Unit, Kyoto University Hospital, Kyoto, Japan

15 Correspondence:

16 Kosuke Fujita

17 Department of Rehabilitation, Nagoya University Hospital

$18 \quad 65$ Tsurumai-cho, Showa-ku, Nagoya, Aichi 466-8560

19 Japan

$21 \quad$ E-mail: fujitak@med.nagoya-u.ac.jp

22 Office phone: +81-75-751-3951

23 Office fax: $+81-75-751-3909$ 
27 Context: A recent review or article reported that thermal agents (TA) or physical activity

28 (PA) can increase range of motion (ROM), and that the combination of TA with stretching is

29 superior to performing stretching only. However, since ROM is affected by the participants' psychological factors, it is questionable whether these studies measured the effect of these

31 intervention on muscle flexibility. By measuring muscle stiffness, we attempted to evaluate

32 the effect these intervention on muscle flexibility.

33 Objective: To compare the individual effects of TA and PA on muscle flexibility, as well as

34 their effectiveness when combined with static stretching (SS).

35 Design: Crossover trial.

36 Setting: University research laboratory.

37 Participants: Fifteen healthy men without a history of orthopedic disease in their lower

38 limbs.

39 Interventions: Fifteen minutes of three different conditions: hot pack as TA, pedaling

40 exercise as PA, and the control group with no TA or PA intervention, followed by three

41 minutes of SS for the hamstrings muscles.

42 Main Outcome Measures: Joint angle and passive torque of the knee during passive

43 elongation were obtained prior to interventions (pre-intervention), following three kinds of 
44 intervention (post-intervention), and after SS (post-SS). From these data, muscle tendon

45 unit (MTU) stiffness of the hamstrings was calculated.

46 Results: Although knee joint ROM increased with both TA and PA $(p<0.05)$, there were no

47 significant differences in MTU stiffness between pre- and post-intervention measurements

48 for either of these interventions (TA, $p=0.477$; $P A, p=0.377$; control, $p=0.388$ ). However,

49 there were similar significant decreases in MTU stiffness between post-intervention and

50 post-SS at all condition $(\mathrm{p}<0.01)$.

51 Conclusions: TA and PA did not decrease MTU stiffness, and combining these

52 interventions with SS did not provide additional decreases in MTU stiffness compared to

53 performing SS alone. 
$\underline{\text { Introduction }}$

The effect of thermal agents on muscle flexibility has been well studied using various

methods of heat interventions ${ }^{1-6}$. Most of these studies have supported the use of heat intervention for increasing range of motion (ROM) 1, 2, 5, 7. A recent review of research with human participants has shown the combination of heat modalities and stretching to be superior to static stretching only for increasing joint ROM but not for lowering the passive stiffness of the muscle at the same joint angle ${ }^{8}$. On the other hand, only a few studies have evaluated the effect of physical activity in improving muscle flexibility ${ }^{9,10}$. Williford et al. reported jogging prior to stretching to be effective in improving muscle flexibility, while is known to be affected by psychological factors, such as pain and stretch tolerance ${ }^{11}$. Recently, measurement of muscle tendon unit stiffness during passive movement has been shown to be a preferred index of muscle flexibility, as this method excludes the effect of psychological factors. The use of muscle tendon unit stiffness, therefore, would be an effective method to differentiate the underlying mechanism of change in joint ROM, whether it is due to decreased muscle stiffness and/or to a change in muscle stretch tolerance. A study by Kubo et al. ${ }^{12}$ did compare the effect of heat and cold water immersion on muscle tendon unit stiffness. However, in their study, Kubo et al. did not examine the effect of physical activity, either alone or in combination with stretching, on muscle tendon unit stiffness. To the best of our knowledge, no study has examined and compared the effectiveness of thermal agent and physical activity interventions, administered prior to static stretching (SS), by using muscle tendon unit stiffness as an index of muscle flexibility. 
The goals of this study were to examine the effect of thermal agent and physical

activity interventions, in combination with SS, on muscle tendon unit stiffness. We

hypothesized that thermal agent and physical activity interventions would contribute an additional effect to SS in decreasing muscle tendon unit stiffness, and that the effect of physical activity would be superior to that of thermal agent.

\section{Methods}

Design

This study used a crossover design. Each subject participated in three sessions, with exposure to all three experimental conditions: the thermal agent condition, in which a hot pack was applied to the hamstring muscles; the physical activity condition, during which subjects performed a pedaling exercise; and the control condition, in which no intervention was provided. Experimental conditions were randomized across subjects to eliminate the effect of order. In each evaluation session, subjects initially underwent either thermal agent, physical activity or rest for the control condition, followed by a period of SS. Measurements of muscle tendon unit stiffness were obtained before thermal agent intervention, physical activity intervention or rest for the control condition (pre-intervention), after the intervention or rest, (post-intervention), and immediately after SS (post-SS). Sessions were held more than two days apart to control for between-condition interactions.

\section{$\underline{\text { Participants }}$}

Fifteen healthy men (mean age, $23.2 \pm 1.9$ years; height, $172.9 \pm 5.2 \mathrm{~cm}$; weight, 
obtained from each participant. This study was approved by the ethics committee of Kyoto university hospital (approval number E1936).

\section{Procedures}

Measurements of muscle tendon unit stiffness and passive torque of the knee were obtained using the Biodex System 4 (Biodex Medical Systems, Inc.; New York, US).

Subjects were seated with a wedge placed under their thigh and another wedge-shaped cushion behind their back, so that the hip and knee joints were flexed to about $120^{\circ}$. The dominant foot was securely attached to the arm of the dynamometer, while the trunk was firmly fixed to the seat with a belt to prevent compensatory pelvic motion (Figure 1). This position was defined as the starting position. Subjects were instructed to remain relaxed during measurements of muscle tendon unit stiffness. To measure the extensibility of the hamstrings, the knee joint was passively extended at a constant velocity of $5 \% \mathrm{~s}$ from the starting position to the final angle, which was defined as the angle just before subjects reported feeling a strong stretching sensation but no pain. The final angle was expressed as the change in knee angle from the starting position. SS in this study was also performed to this final angle, which was individually determined. Muscle tendon unit stiffness was calculated as the change in passive torque divided by the change in joint angle and, therefore, quantified by the slope of the torque-angle curve over the last $10 \%$ of passive knee extension ${ }^{13}$. Passive torque at the final angle was used as an index of stretch tolerance, reflecting the psychological factors (Figure 2).

For the thermal agent condition, subjects were required to lie prone for $15 \mathrm{~min}$, with a hot pack applied to their hamstrings. The duration of 15 min was based on previous research ${ }^{7,14}$, which we consider to be a typical duration in a clinical setting. Each hot pack (S-PACK: SAKAI Medical Co., Ltd.) was heated to $80^{\circ} \mathrm{C}$ in a hydro-collator tank 
130 (PACKWARMER CL-15: SAKAI Medical Co., Ltd.) and wrapped in double layers with a 131 thick towel. For the physical activity condition, subjects performed a pedaling exercise for 13215 min, using an Aerobike 75XLIII (Konami Sports \& Life Co., Ltd.). Exercise intensity was 133 controlled by cadence or load adjustment, which was self-selected by subjects to maintain 134 their heart rate at $60 \%$ of their maximum voluntary exercise capacity, calculated using the 135 Karvonen formula. Subjects increased their heart rate up to their target heart rate over the 136 first 3 min of pedaling, and maintained this target rate for $12 \mathrm{~min}$. In the control condition, 137 subjects were required to lie prone on the bed for $15 \mathrm{~min}$. Immediately after the post138 intervention measurement, a 3-min SS was performed at the final angle measured on the 139 dynamometer. The post-SS measurement was obtained at the end of the stretching period. 140 The time between the intervention and SS and that between the SS and post-SS 141 measurement was minimized to $45 \mathrm{~s}$ and $15 \mathrm{~s}$, respectively.

\section{Statistical Analysis}

SPSS ver. 17 (IBM Japan Ltd.) was used for statistical analysis. A two-way repeated-measures analysis of variance (ANOVA, condition $\times$ period) was used to evaluate differences in final angle, muscle tendon unit stiffness, and passive knee joint torque at the final angle. Differences were considered statistically significant at values of $P<0.05$. When a significant interaction or main effect of the condition was identified, the item was assessed for significant differences using a t-test, with Bonferroni adjustment, as a post-hoc test. A priori power analysis indicated that a sample size of 12 subjects for each group would be required to obtain $80 \%$ power, if we estimated an F-statistic of 0.4 for a large effect size with $\alpha=0.05$. 
Two-way ANOVA identified no significant interaction effects between condition and period $(P=0.97, F=0.14)$ on muscle tendon unit stiffness, but did indicate a significant main effect of period $(P<0.05)$. In all conditions, the post-hoc test for period indicated no

159 significant difference between pre- and post-intervention measurements $(P>0.05)$;

160 however, muscle tendon unit stiffness post-SS was significantly lower than post-intervention $161 \quad(\mathrm{P}<0.05)($ Table 1$)$.

Final Angle

Two-way ANOVA indicated a significant interaction effect between condition and period $(P=0.03, F=2.71)$ on the final angle. In addition, a significant main effect of both condition and period was identified $(P<0.05)$. Post-hoc testing of the period indicated postintervention final angle to be significantly larger than the angle measured pre-intervention, for both the thermal agent and physical activity conditions $(P<0.05)$. In addition, the final angle measured post-SS was significantly larger than the final angle measured postintervention for both thermal agent and physical activity $(P<0.05)$. For the control condition, the final angle post-SS was significantly larger than the post-intervention angle $(P$ $172<0.05)$, whereas no significant difference in final angle was identified between pre- and post-intervention measures $(P>0.05)$. Post-hoc testing for the effect of condition indicated

174 that post-intervention final angles for both thermal agent and physical activity were 175 significantly larger than angles for the control condition $(P<0.05)$.

\section{Passive Torque at the Final Angle}

Two-way ANOVA indicated a significant interaction between condition and period ( $P$ $179<0.01, F=5.98$ ) on passive torque at the final angle. In addition, a significant main effect 
180

181

was identified for both condition and period $(P<0.05)$. Post-hoc testing of the period indicated that the post-intervention passive torque at the final angle for the thermal agent condition was significantly higher than the values measured in the pre-intervention $(P<$ 0.05), and post-SS was significantly higher than post-intervention $(P<0.05)$. On the other hand, for the physical activity condition, passive torque at the final angle was significantly higher post-intervention than pre-intervention $(P<0.05)$, but there was no significant difference between the post-intervention value and values measured post-SS $(P>0.05)$. For the control condition, there was no significant difference between pre- and postintervention measures $(P>0.05)$. However, passive torque at the final angle post-SS was significantly higher than at post-intervention $(P<0.05)$. Post-hoc testing of the condition indicated that post-intervention measures of passive torque at the final angle for the thermal agent and physical activity conditions were significantly higher than values for the control condition $(P<0.05)$.

$$
\begin{aligned}
& \text { Discussion } \\
& \text { This study had two objectives. The first was to compare the effect of thermal agent } \\
& \text { and physical activity on hamstring muscle flexibility, using muscle tendon unit stiffness as } \\
& \text { an outcome measure. The second was to examine whether thermal agent or physical } \\
& \text { activity, performed before SS, was more effective at increasing hamstring muscle flexibility } \\
& \text { than SS alone. To our knowledge, this is the first study to compare the effect of thermal } \\
& \text { agent and physical activity interventions on muscle tendon unit stiffness, and their } \\
& \text { combined effects with SS, on muscle tendon unit stiffness. }
\end{aligned}
$$


205 changes in muscle tendon unit stiffness measured pre- and post-intervention, in spite of 206 improvements in knee ROM for both interventions. The improvement in ROM is consistent 207 with previous studies having examined the effect of heat on muscle flexibility $1,2,5,7$. Our 208 study also indicated that there is no difference between thermal agent and physical activity 209 on either ROM or muscle stiffness, post-intervention. Demura et al. (2006) have shown that 210 heat and light exercise (bicycle ergometer) are equally effective and superior to placebo for 211 increasing ROM, which is consistent with our results for ROM. The absence of a significant 212 change in muscle tendon unit stiffness with either the thermal agent or physical activity 213 intervention is in agreement with results from Kubo et al. (2005), who reported that 30 min 214 of hot water immersion yielded no change in the mechanical property of the muscle and 215 tendon. Although in vitro studies ${ }^{14,15}$ have indicated that flexibility can be increased by heat 216 stimulation, there seems to be discrepancy in soft tissue temperatures between these 217 studies and our study. In these studies, the soft tissue was warmed to about $45^{\circ} \mathrm{C}$.

218 According to a previous in vivo study, which reported that a 15 min of hot pack can increase 219 the soft tissue to only $36-38^{\circ} \mathrm{C}^{16}$, the change in the soft tissue temperature in the thermal 220 agent condition in our study might have been much lower compared to the previous in vitro studies, which may have produced different effects on muscle tendon unit stiffness.

The duration of applying thermal agent and physical activity intervention was set as 15 min, following a clinical standard and previous research. In previous research in vivo, the application time of hot pack was set at about $15^{7,16}-20^{1} \mathrm{~min}$. Draper et al (1998) described that the application of a hot pack for 15 min raised the body temperature by $3.83{ }^{\circ} \mathrm{C}$ at a depth of $1 \mathrm{~cm}$ and by $0.74{ }^{\circ} \mathrm{C}$ at a depth of $3 \mathrm{~cm}$. Therefore, it is reasonable to assume that the soft tissue temperature in this study was raised to the same extent. However, there is an evident discrepancy in estimates of the tissue temperature attained between research in 
vivo and in vitro, which could affect muscle tendon unit stiffness.

Regarding the combination of thermal agent and physical activity intervention with SS, all three conditions, including the control, showed significant decreases in muscle tendon unit stiffness after SS. Moreover, there were no differences between the three conditions, indicating that the combination of thermal agent or physical activity with SS might have no additional effect on muscle tendon unit stiffness compared to SS alone. Again, knee joint ROM increased in all three conditions, compared to both pre- and postintervention measurements, and there were no significant differences between the three intervention groups.

These results indicate that thermal agent and physical activity are both effective in increasing joint ROM but not in altering muscle tendon unit stiffness. This indicates that improvements in ROM are likely due to changes in sensory perception or stretch tolerance. The combination of thermal agent and physical activity with SS had no advantage compared to SS alone in improving either ROM or muscle tendon unit stiffness, which contradicts our a priori hypothesis. The result from our study contradict findings in a previous review evaluating the combination of heat modalities (hot pack, ultrasound and hot water immersion, duration 8 to $20 \mathrm{~min}$ ) and stretching ${ }^{8}$, which reported that the combination of heat and stretching was superior to stretching alone. However, this contradiction in findings seems reasonable when we consider that most of the studies included in this review used ROM as a measure of joint flexibility, instead of muscle tendon unit stiffness as used in our study. 
255 index of stretch tolerance, with an increase in this index indicating alteration in subjects' sensory perception of muscle elongation ${ }^{17-19}$. It is well known that muscle warming modifies this sensory perception. In addition to muscle warming intervention, previous studies have reported that SS provides a 'dulling' of this sensory perception, which may indicate that centripetal input from the muscle and joint, resulting from SS, alters the input 260 from the nociceptive nerve endings and may increase the pain sensation threshold ${ }^{20-22}$. In this study, such changes in threshold may have interacted to increase the passive torque at 262 the final angle after muscle warming intervention and/or SS. The absence of an increase in 263 passive torque at final angle with combined physical activity and SS may be explained by a 264 ceiling effect of physical activity on the modulation of this pain mechanism, presuming that pain threshold differ among intervention methods, and modification of sensation has an end point. The effect of performing physical activity may have been large enough to reach the end point that no additional effect was possible by adding SS. Considering these results, the combined use of muscle warming and SS may have no additional effect on muscle tendon unit stiffness measured by the passive torque at the final joint angle compared to SS only. This theory is based on the hypothesis that modifying sensation has end point which needs further inspection.

274 both thermal agent and physical activity and only one duration for the heat application.

275 Therefore, it is unknown whether these results apply to other types of thermal agent and physical activity interventions or to longer/shorter heat application times. In addition, there are no data concerning tissue temperature in this study. Therefore, the effect of the interventions on tissue temperature can only be assumed based on previous studies, which used similar protocols._In addition, we did not examine any effects that may have occurred 
280 in time after the intervention.

This result suggests that in the purpose of increasing flexibility, there is no additional

benefit on applying thermal agent or physical activity preceding SS in healthy subjects.

However, if the patient is too sensitive to perform SS adequately for some reason, it may be

a good solution to modify sensation by conducting these interventions prior to performing

solution to apply thermal interventions.

\section{$\underline{\text { Conclusion }}$}

This study showed that, though thermal agents and physical activity are effective in increasing ROM, this effect is due to change in sensory perception and not to change in

muscle tendon unit stiffness. Our results also indicate that performing thermal agent and

physical activity intervention prior to SS is not effective in augmenting the effect of agents, physical activity and stretching in clinical settings.

\section{$\underline{\text { References }}$}

1. Cosgray NA, Lawrance SE, Mestrich JD, Martin SE, Whalen RL. Effect of heat modalities on hamstring 304 length: A comparison of pneumatherm, moist heat pack, and a control. J Orthop Sports Phys Ther. 305 2004;34(7):377-384.

306 2. Funk D, Swank AM, Adams KJ, Treolo D. Efficacy of moist heat pack application over static stretching 
on hamstring flexibility. J Strength Cond Res. 2001;15(1):123-126.

308 3. Lentell G, Hetherington $T$, Eagan J, Morgan M. The use of thermal agents to influence the 309 effectiveness of a low-load prolonged stretch. J Orthop Sports Phys Ther. 1992;16(5):200-207.

310 4. Benoit TG, Martin DE, Perrin DH. Hot and cold whirlpool treatments and knee joint laxity. J Athl Train. 311 1996;31(3):242-244.

$312 \quad 5 . \quad$ Sakulsriprasert P, Vongsirinavarat M, Thammajaree C, Khoblueng D, Sunthornwiriyawong K. Effect of 313 superficial heating duration on plantarflexor extensibility. Songklanagarind Med J. 2011;28(6):295-304.

314 6. Sawyer PC, Uhl TL, Mattacola CG, Johnson DL, Yates JW. Effects of moist heat on hamstring flexibility 315 and muscle temperature. J Strength Cond Res. 2003;17(2):285-290.

$3167 . \quad$ Robertson VJ, Ward AR, Jung P. The effect of heat on tissue extensibility: A comparison of deep and 317 superficial heating. Arch Phys Med Rehabil. 2005;86(4):819-825.

318 8. Bleakley CM, Costello JT. Do thermal agents affect range of movement and mechanical properties in 319 soft tissues? A systematic review. Arch Phys Med Rehabil. 2013;94(1):149-163.

320 9. Williford HN, East JB, Smith FH, Burry LA. Evaluation of warm-up for improvement in flexibility. Am J 321 Sports Med. 1986;14(4):316-319.

322 10. Demura S, Noguchi T, Matsuzawa J. Comparison in the effect of linear polarized near-infrared light 323 irradiation and light exercise on shoulder joint flexibility. Clin J Sport Med. 2006;16(4):293-297.

324 11. McHugh MP, Kremenic IJ, Fox MB, Gleim GW. The role of mechanical and neural restraints to joint 325 range of motion during passive stretch. Med Sci Sports Exerc. 1998;30(6):928-932.

326 12. Kubo K, Kanehisa H, Fukunaga T. Effects of cold and hot water immersion on the mechanical 327 properties of human muscle and tendon in vivo. Clin Biomech (Bristol, Avon). 2005;20(3):291-300.

328 13. Reid DA, McNair PJ. Effects of an acute hamstring stretch in people with and without osteoarthritis of 329 the knee. Physiotherapy. 2010;96(1):14-21.

330 14. Bass CR, Planchak CJ, Salzar RS, Lucas SR, Rafaels KA, et al. The temperature-dependent 331 viscoelasticity of porcine lumbar spine ligaments. Spine (Phila Pa 1976). 2007;32(16):E436-442.

332 15. Wang JC, Kabo JM, Tsou PM, Halevi L, Shamie AN. The effect of uniform heating on the 333 biomechanical properties of the intervertebral disc in a porcine model. Spine J. 2005;5(1):64-70.

334 16. Draper DO, Harris ST, Schulthies S, Durrant E, Knight KL, et al. Hot-pack and 1-mhz ultrasound 335 treatments have an additive effect on muscle temperature increase. J Athl Train. 1998;33(1):21-24.

336 17. Halbertsma JP, van Bolhuis AI, Goeken LN. Sport stretching: Effect on passive muscle stiffness of 337 short hamstrings. Arch Phys Med Rehabil. 1996;77(7):688-692.

338 18. Muir IW, Chesworth BM, Vandervoort AA. Effect of a static calf-stretching exercise on the resistive 339 torque during passive ankle dorsiflexion in healthy subjects. J Orthop Sports Phys Ther. 1999;29(2):106-113; 340 discussion 114-105.

341 19. Magnusson SP, Aagard P, Simonsen E, Bojsen-Moller F. A biomechanical evaluation of cyclic and 342 static stretch in human skeletal muscle. Int J Sports Med. 1998;19(5):310-316.

343 20. Azevedo DC, Melo RM, Alves Correa RV, Chalmers G. Uninvolved versus target muscle contraction 344 during contract: Relax proprioceptive neuromuscular facilitation stretching. Phys Ther Sport. 2011;12(3):117- 
345121.

346 21. Magnusson SP, Simonsen EB, Aagaard P, Dyhre-Poulsen P, McHugh MP, et al. Mechanical and 347 physical responses to stretching with and without preisometric contraction in human skeletal muscle. Arch Phys 348 Med Rehabil. 1996;77(4):373-378.

349 22. Law RY, Harvey LA, Nicholas MK, Tonkin L, De Sousa M, et al. Stretch exercises increase tolerance 350 to stretch in patients with chronic musculoskeletal pain: A randomized controlled trial. Phys Ther. $351 \quad$ 2009;89(10):1016-1026. 
354 Table 1. Stiffness, final angle, and passive torque at the final angle

355

\begin{tabular}{|c|c|c|c|c|c|c|c|c|c|}
\hline & \multicolumn{3}{|c|}{ Stiffness $\left(\mathrm{Nm} /{ }^{\circ}\right)$} & \multicolumn{3}{|c|}{ Final Angle $\left({ }^{\circ}\right)$} & \multicolumn{3}{|c|}{ Passive Torque at the Final Angle (Nm) } \\
\hline & Pre & Post & Post-SS & Pre & Post & Post-SS & Pre & Post & Post-SS \\
\hline Control & $1.18 \pm 0.22$ & $1.18 \pm 0.23$ & $0.92 \pm 0.19^{* *},+\dagger$ & $48.27 \pm 11.29$ & $44.39 \pm 10.62$ & $64.70 \pm 9.68^{* *+\dagger}$ & $45.12 \pm 7.65$ & $39.38 \pm 4.91$ & $52.72 \pm 9.54^{* *},+\dagger$ \\
\hline $\begin{array}{l}\text { Thermal } \\
\text { Agent }\end{array}$ & $1.19 \pm 0.16$ & $1.22 \pm 0.18$ & $0.88 \pm 0.26^{* *+, \dagger}$ & $49.48 \pm 9.20$ & $59.29 \pm 12.15^{\star}$, & $72.49 \pm 11.66^{* *+1 \dagger}$ & $43.57 \pm 7.27$ & $51.95 \pm 7.55^{\star}$ & $59.17 \pm 8.96$ \\
\hline $\begin{array}{l}\text { Physical } \\
\text { Activity }\end{array}$ & $1.17 \pm 0.19$ & $1.19 \pm 0.18$ & $0.86 \pm 0.13^{* *}, \dagger+$ & $47.11 \pm 13.35$ & $60.72 \pm 9.84^{\star *}$, & $72.14 \pm 8.79^{* *}, \dagger$ & $44.37 \pm 7.03$ & $56.05 \pm 7.13^{\star \star}$, & $58.89 \pm 9.38^{\star \star}$ \\
\hline
\end{tabular}

Abbreviations: Pre, preintervention; Post, postintervention; SS, static stretching

${ }^{*} \mathrm{P}<.05$ versus pre.

${ }^{*} \mathrm{P}<.01$ versus pre.

${ }^{\dagger} \mathrm{P}<.05$ versus postintervention.

${ }^{\mathrm{t}} \mathrm{P}<.01$ versus postintervention.

${ }_{\ddagger \neq} \mathrm{P}<.01$ versus control in the same period 
358 Figure1. Measurement position for stretch maneuver: (1) wedge under the thigh; (2) wedge359 shaped cushion behind the back; (3) belt stabilizing the trunk; (4) attachment to the load 360 cell.

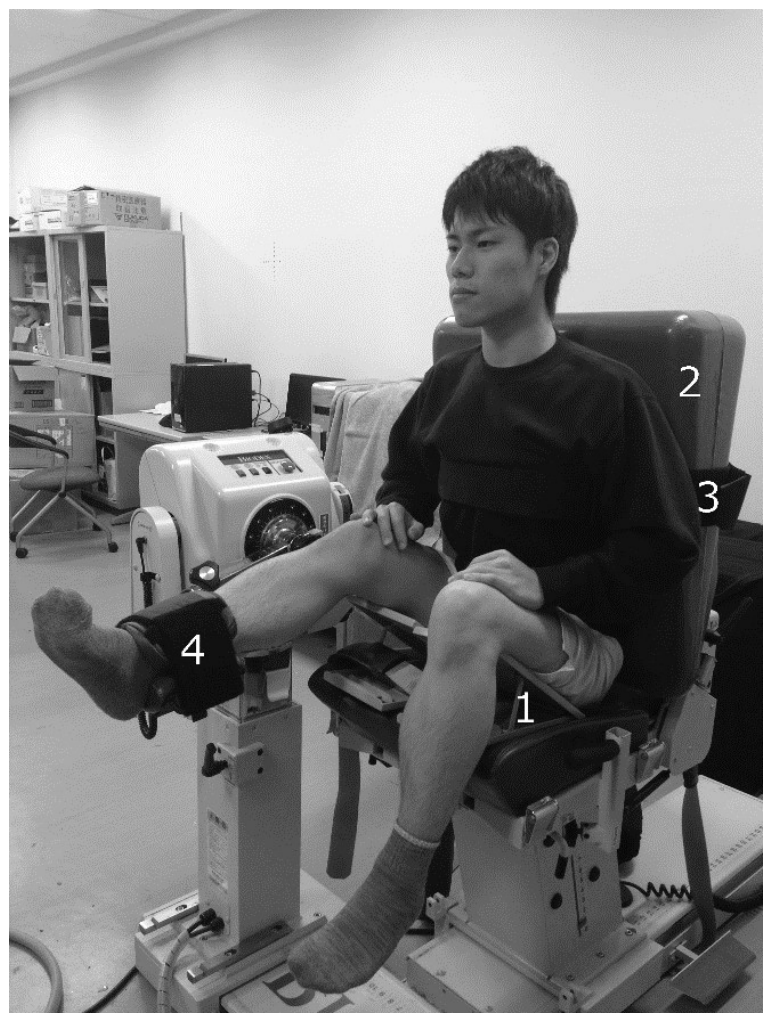


363 Figure2. Measurement indices used in this study showing passive torque during passive 364 extension of the knee.

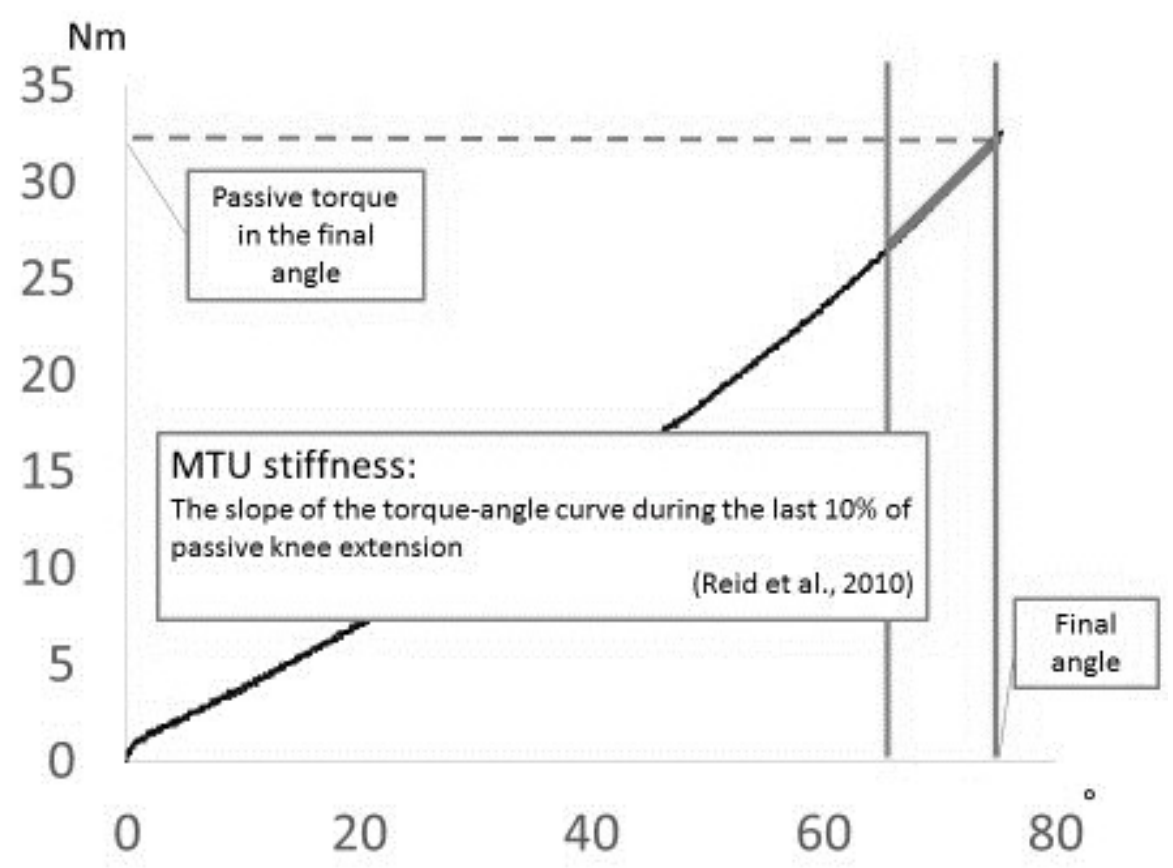

